\title{
Quaderni
}

QUADERNI Communication, technologies, pouvoir

\author{
71 | Hiver 2009-2010 \\ Le changement climatique : les résistances à \\ l'adaptation
}

\section{Voyages en France : recherches sur l'adaptation au réchauffement climatique}

\section{Lucien Sfez}

\section{(2) OpenEdition \\ Journals}

Édition électronique

URL : http://journals.openedition.org/quaderni/529

DOI : 10.4000/quaderni.529

ISSN : 2105-2956

Éditeur

Les éditions de la Maison des sciences de l'Homme

Édition imprimée

Date de publication : 5 janvier 2010

Pagination : 61-74

\section{Référence électronique}

Lucien Sfez, « Voyages en France : recherches sur l'adaptation au réchauffement climatique », Quaderni [En ligne], 71 | Hiver 2009-2010, mis en ligne le 05 janvier 2012, consulté le 19 avril 2019. URL : http://journals.openedition.org/quaderni/529 ; DOI : 10.4000/quaderni.529 


\section{$D$ ossier}

\section{voyages en France :}

recherches sur l'adaptation au réchauffement climatique À la demande de l'A.D.E.M.E. nous avons procédé à quatre enquêtes sur l'adaptation des populations au réchauffement climatique ${ }^{1}$. Il est hors de question ici d'en présenter les éléments de façon autre que sommaire.

On décrira successivement les terrains (Première partie) et nos conclusions plus théoriques (Deuxième partie), sachant que pour une analyse exhaustive, on se reportera aux rapports rendus à l'A.D.E.M.E. ${ }^{2}$

\section{Première partie : les terrains}

Ils sont au nombre de quatre. De la moyenne montagne des Alpes du Sud (A) à la Camargue (B), en passant par la Champagne (C) et jusqu'au Bordelais (D) .

Lucien

Sfez

Professeur émérite Université de Paris 1 Panthéon Sorbonne

\section{A. Les Alpes du Sud}

Nous avons commencé, dans l'hiver 2004/2005, par travailler sur deux villages de moyenne montagne dans les Alpes du Sud, tous deux dépourvus de neige en pleine saison et à $8 \mathrm{Km}$ l'un de l'autre.

Le premier village, Le Sauze, est dominé par une famille, propriétaire de la station de ski, de nombreux terrains et immeubles, famille descendante du maire qui fonda la station de ski dans les années 1930. Ici ce serait peu de dire que l'on est resté sourd à la question du réchauffement. Cette question n'existe pas, et ceux qui viennent enquêter sur la prise de conscience du phénomène sont agressés (au moyen de la voix) par le propriétaire de la station et ce, en public. Ils viennent de la part de 
l'A.D.E.M.E., et avec - circonstance aggravante - une casquette de professeur à la Sorbonne : ce ne sont pas de bonnes références !

Le second village, dans la même vallée de l'Ubaye, Pra-Lou, n'est pas une propriété familiale ; il est né dans les années 1970 du temps de Georges Pompidou. C'est un village « industriel » dont l'industrie est tout entière tournée vers l'exploitation de la neige avec de nombreux immeubles d'habitation assez froids d'apparence et peu amènes, surtout quand on traverse le bourg totalement vide pour cause d'absence de neige en saison et en plein jour ! Pourtant, là, les conseillers municipaux de toutes tendances, des hôteliers et restaurateurs nous reçurent avec sympathie, admirent la réalité du phénomène, cherchèrent avec nous les moyens d'y parer, jusqu'à, pour certains, imaginer de partir en Pologne pour trouver la neige, base de leur savoir-faire économique. Deux attitudes contrastées. Pourquoi ?

C'est que les premiers font reposer toute leur mythologie active sur le passé : l'ancien maire fondateur a sa statue, énorme, qui domine l'entrée du village, tandis que les seconds n'ont pas cette mémoire-là et gagnent seulement leur vie. Nous verrons dans la deuxième partie les conclusions que nous pourrons tirer d'une certaine forme de mémoire, celle qui bloque .

\section{B. La Camargue}

Un an plus tard, en 2005/2006, nous sommes partis travailler sur la Camargue, dont les documents de l'O.N.E.R.C. et les travaux des géographes décrivaient les difficultés : montée de 2 millimètres par an du niveau de la mer depuis le début du $\mathrm{XX}^{\mathrm{e}}$ siècle, par suite de la dilatation de la mer, dûe au réchauffement, et à la fonte des glaciers ; disparition progressive des alluvions issus du Rhône en raison des barrages d'E.D.F., ceci empêchant l'existence de sable et de plages, sans compter les courants qui emmènent au loin le peu d'alluvions qui reste ; pluies diluviennes, dites " cévenoles », dont la concentration, forte en certaines saisons, entraîne souvent des inondations, et qui, de l'avis de certains spécialistes, s'explique par le réchauffement climatique.

La Camargue est un grand territoire, d'une grande diversité et où chaque lieu a sa spécificité. Supposez un triangle dont le sommet serait Arles, dont le côté gauche irait d'Arles aux Saintes-Maries-de-la--Mer et dont le côté droit irait d'Arles à Salin-de-Giraud. Et ici, nous ne vous parlons pas, pour simplifier, de la « grande Camargue », triangle plus grand dont le côté gauche irait d'Arles à Aigues-Mortes...

Donc, le petit triangle seulement : Arles toujours au sommet, menacée par les inondations du Rhône, ville la plus importante et la plus peuplée de la région, dirigée par une coalition d'Union de la gauche, écologistes inclus, avec un maireadjoint écologiste, médecin à l'hôpital d'Arles, un maire-adjoint socialiste, instituteur de son état et un maire communiste, ancien chercheur au C.N.R.S. Il est bien évident ici que l'accueil fut agréable, avec une grande attention aux problèmes soulevés, que l'on ne se permit pas de nier les questions écologique, le discours des savants du G.I.E.C., les difficultés venant des inondations issues du Rhône et les travaux de 
fond destinés à y faire face. De la même façon, nos interlocuteurs affichèrent leur soutien au Parc Naturel Régional de Camargue, que le maire d'Arles n'hésita pas à appeler « le parlement de la Camargue ». Le changement climatique était au rang des problèmes importants de cette équipe, avec cette nuance que la nature est là pour l'homme et non pas l'inverse, aux dires du maire. Mais nous n'étions pas là pour vérifier la rigueur du dogme écologique...

Peu différent fut l'accueil de Salin-de-Giraud : pour autant que l'accueil était celui de la mairie (Salin-de-Giraud relève de la mairie d'Arles et le responsable de Salin-de-Giraud était l'élu socialiste précité), la réception fut excellente et l'élu socialiste convoqua même pour nous un Conseil de village autour de notre questionnaire, afin de déterminer si, oui ou non, il y avait changement climatique.

Mais la réception à la Compagnie des Salins du Midi, sise à Salin-de-Giraud fut plus ambiguë : empreinte de défiance, avec un entretien raccourci par suite d'une urgence inventée, un mensonge délibéré sur la situation économique difficile des Salins, ce qu'on nia éperdument (mais le nombre d'emplois sera bientôt drastiquement réduit, ce qu'on voulait cacher) et pour couronner le tout, une certitude de croyant sur les ouvrages de la Compagnie du Midi, destinés à contenir la mer : ils étaient parfaits, disait-on, coûtaient fort cher et on ne demandait rien à l'État. Que de vertus ! Mais il faut savoir aussi que les terrains sur lesquels la Compagnie a son activité et dont elle est propriétaire sont installés en bord de mer et valent des milliards... Oui, les ouvrages sont de bonne qualité, non, les travaux ne coûtent rien à l'État, mais tout ceci a un prix que le Conservatoire du Littoral aura à payer s'il veut maintenir le paysage en l'état. Et si le Conservatoire ne veut pas acheter, ces terrains à bâtir seront vendus à très haut prix à des spéculateurs fonciers. Tout ceci fut non-dit, mais bien connu à l'avance par les chercheurs.

Aux Saintes-Maries-de-la-Mer, l'accueil fut franchement plus mauvais : le député-maire de l'époque, charcutier de son état, nous fit répondre que ça ne l'intéressait pas (comme on dit aux vendeurs d'aspirateurs). Il était connu jusqu'à Marseille pour son hostilité à toute étude, fûtelle celle du B.R.G.M. À quoi bon des études, lui est élu et il sait. Il faut faire plus de digues etc. Le maire-adjoint, médecin, nous reçut avec courtoisie, mais nous indiqua vite que toute cette affaire de réchauffement était une lubie de scientifiques et qu'il ne fallait rien en croire. Tandis que le directeur de la capitainerie du port, fonctionnaire de la mairie, et à ce titre sous les ordres du maire, nous exprima de son côté toute sa haine à l'égard des médias qui ne disent pas la vérité, qui grossissent démesurément les difficultés et empêchent la malheureuse population de plagistes, de commerçants, de restaurateurs d'exercer honorablement son activité en décourageant les vacanciers. C'était en effet l'électorat de la mairie, très hostile à toute manifestation de la vérité. Il était exclu de reconnaître que la mer montait, ou que le Rhône pouvait être menaçant... Par ailleurs, le Parc Naturel Régional de Camargue se mêle de ce qui ne le regarde pas et n'est pas et ne doit pas être l'instance centrale de la Camargue. Non, il n'y a pas de danger climatique, laissez-nous vivre comme nous voulons, quitte à demander à l'État 
et à la Région des subventions pour améliorer les équipements protecteurs. Cette attitude proprement passéiste, défensive et pathologique fut rémunérée bientôt par un succès de la même équipe à la mairie des Saintes-Maries-de-la-Mer aux élections municipales, après un échec aux législatives.

Mais à Arles et au Parc Naturel Régional, on nous a beaucoup parlé de l'oubli par les habitants de la culture du risque, puisque les habitants continuent à vouloir s'installer dans des territoires inondables. Il faudrait faire comme les Romains qui savaient, eux... Nous verrons ce qu'il convient de penser de cette suggestion hasardeuse dans la deuxième partie .

\section{La Champagne}

En 2006/2007, nous avons jeté notre dévolu sur les effets du réchauffement climatique en Champagne, sachant que le réchauffement faisait déjà sentir ses effets sur le monde viticole.

Nous savions au départ que l'acteur principal était le Comité Interprofessionnel des vins de Champagne (C.I.V.C.), qu'il nous fallait passer par lui et que mieux encore, rien ne pouvait être entrepris sans lui. Nous avons alors pris contact avec le directeur des services techniques, pièce essentielle du dispositif : c'était en mai 2006. Il ne put nous répondre qu'en septembre. Aucune explication à ce retard, sinon qu'il était très occupé. Un début d'éclaircissement nous fut donné par son adjoint en octobre : «Dans le fond, nous dit-il, nous avons compris que vous et nous étions dans le même combat ». En effet, envoyés par l'A.D.E.M.E., instance en liaison constante avec l'expert en bilan carbone du C.I.V.C., nousmêmes universitaires, nous ne pouvions être des ennemis. D'ailleurs, ce bilan carbone entrepris par le C.I.V.C., était la raison essentielle de notre enquête : si la filière professionnelle du Champagne avait engagé le processus du bilan carbone, n'était-ce pas l'indice d'une sensibilité écologique? Et jusqu'à quel point? Or nous rappelons que l'intérêt de l'A.D.E.M.E. pour nos recherches était dominé par une question : comment mobiliser des territoires (la Camargue), ou des filières (la neige) sur la question du réchauffement climatique? Après nos difficultés dans un village des Alpes du Sud etdans une localité en Camargue, il était temps de choisir une filière a priori plus favorable, plus réceptive.

À partir du moment où le C.I.V.C. nous ouvrit ses portes, tout devint facile, grâce à la liste d'adresses qui nous avait été communiquée par le service technique. Tous les rendez-vous furent pris avec une grande rapidité. Une grande liberté aussi, car lorsque nous cherchâmes à rencontrer des vignerons, nous eûmes de nombreux rendez-vous avec d'autres que ceux proposés par le C.I.V.C. et en particulier avec des vignerons bios.

Une fois comprise, la situation était fort simple : toute la vie professionnelle de la Champagne est dominée par la tension, qui fut lutte (avec des morts) au début du $\mathrm{XX}^{\mathrm{e}}$ siècle et qui est devenue dialogue institutionnalisé au sein du C.I.V.C. entre deux familles, les vignerons et les négociants, ceux-ci, cherchant à acheter le raisin ou le vin au plus bas niveau possible, ceux-là cherchant à le vendre au plus haut prix qu'il est possible. Discussions secrètes et d'autant 
plus secrètes que toute manipulation préalable sur les prix est interdite par les Communautés Européennes. Mais tant qu'on ne peut rien prouver... Par ailleurs, la convergence des deux familles s'élargit, bien sûr, à la technique viticole et vinicole : le service technique est puissant, composé de plus de cent personnes, il expérimente lui-même toutes les innovations et sa direction est reconnue dans les congrès scientifiques. C'est cette même direction, de tendance assez philo écologique, qui a fait appel à l'expertise de M. Jancovici et à son bilan carbone. C'est dire que le C.I.V.C., qui s'intitule volontiers " ministère de défense de la Champagne " est l'instance typique de sensibilisation et de mobilisation de la profession en matière de réchauffement climatique. Cette instance, qui est le fruit de d'une histoire tendue entre les deux familles, s'est coalisée et structurée de façon solide et fait, peut-on avancer sans jeu de mots, la pluie et le beau temps en Champagne. Nous avons d'ailleurs tiré une conclusion conceptuelle de cet aspect fondamental : la nécessité d'une liaison structurée, comme nous le développerons dans la deuxième partie.

Autre aspect, il n'y a pas de crise économique du champagne, car le réchauffement, sensible, entraîne une amélioration de la quantité produite et de la qualité et que cela facilite tous les investissements dispendieux, voire les petits sacrifices qu'entrâne une politique écologique active.

Donc interprofession structurée et économie florissante : deux caractéristiques qui expliquent la réceptivité du Champagne aux innovations écologiques. Deux caractéristiques qui n'existent pas dans le cas du bordeaux.

\section{Le Bordelais}

Voilà pourquoi, nous nous sommes proposés de poursuivre l'enquête sur la viticulture dans un milieu moins favorable, avec une interprofession moins unie, moins structurée, et travaillée depuis 2001 par une crise économique qui s'estompe peu à peu certes, mais qui n'a pas disparu, loin de là. Nous sommes donc partis en février 2008 dans le Bordelais et nous avons rendu notre rapport à l'A.D.E.M.E. en juin 2008.

Deux différences ici d'avec la Champagne :

1. Le Conseil interprofessionnel du vin de Bordeaux (C.I.V.B.) n'est pas le Comité interprofessionnel du vin de Champagne (C.I.V.C.) Toujours deux familles, négociants et vignerons, mais qui ne parviennent pas à se mettre d'accord, que ce soit sur les politiques économiques ou sur les questions techniques. Dans certains cas, les désaccords sont tels que les décisions ne sont pas prises. Exemple parmi d'autres : le C.I.V.B, sur la proposition de ses services techniques et avec l'appui de son directeur général, a voulu imiter le C.I.V.C. en faisant appel au bilan carbone du même M. Jancovici. Bonne idée, sans conteste, pour conscientiser les esprits subtils et fuyants du Bordelais : peu, parmi les viticulteurs, sont conscients du phénomène, à l'exception, bien sûr, des grands crus classés qui prennent des mesures précises de température et de pluviométrie dans leurs propriétés. Bonne idée, mais dans l'interview qu'il nous a donnée, 
le président du C.I.V.B., après avoir entendu la conférence de M. Jancovici a cru bon de nous dire qu'il n'en croyait pas un mot, que le conférencier avait choisi de mauvais chiffres en excluant les bons, de façon à plus facilement convaincre. Nous avons ici presque entendu qu'il s'agissait là d'une tromperie délibérée... Si le président du C.I.V.B. en est là, comment peut-on passer par le C.I.V.B. pour convaincre les viticulteurs qui doutent, et doutent d'autant plus que, climat Atlantique aidant, les données climatiques sont à la fois certaines sur le plan scientifique et peu évidentes à percevoir ? Circonstance aggravante : le réchauffement climatique, tout comme en Champagne a des effets bénéfiques sur les quantités produites et même sur la qualité. C'est à peine si l'on concède que l'année 2003, excellente, n'a pas donné des vins de garde.

2. Deuxième différence d'avec la Champagne : les préoccupations sont ailleurs. Les bordeaux supérieurs et les petits bordeaux se vendent mal et la crise pour eux est toujours là, le Conseil d'État a annulé le classement des crus bourgeois en 2007, la réforme des A.O.C. est toujours en instance, voilà tout un ensemble de phénomènes qui ne contribuent pas, tant s'en faut, à rendre consciente la profession de l'urgence climatique. Les autres urgences occupent à elles seules le centre du tableau. Il faudra sans doute dépenser des trésors d'ingéniosité pour mobiliser ce milieu girondin, qui se méfie toujours beaucoup des modes parisiennes, sur les questions du climat.

Nous avons pu à ce sujet tirer quelques conclusions théoriques sur la question de l'identité, à partir de l'expérience bordelaise, comme nous le verrons dans la deuxième partie. Au total, quelles premières conclusions pouvonsnous tirer de ces quatre terrains d'un point de vue strictement empirique?

La perception du changement climatique est très inégale. Ou bien on ne peut nier le changement (cas de la disparition de la neige en moyenne montagne) et dans ce cas on peut y remédier par la technique qui aggrave le mal (canons à neige) ou par une adaptation plus raisonnée vers d'autres activités ; ou bien on refuse d'entendre en niant le phénomène (cas des Saintes-Mariesde-la-Mer) ; ou bien on accepte la réalité du phénomène pour s'en réjouir comme en Champagne, tout en préparant l'avenir grâce au C.I.V.C. ; ou bien on ne perçoit pas bien le phénomène pour des raisons objectives (climat atlantique dans le Bordelais), pour des raisons tenant à de plus grandes urgences (crise économique et réformes de l'A.O.C. dans le Bordelais) avec une instance de régulation assez faible (cas du C.I.V.B. bordelais encore). Il y a donc des circonstances favorables à la prise de conscience et d'autres à la mobilisations des esprits. Ce ne sont pas les mêmes, ce qui nous intrigue. Tentons maintenant d'éclaircir ces points.

\section{Deuxième partie : quelques conclusions théoriques}

Où nous verrons ici que, commençant par la mémoire dans le premier terrain, nous finirons par la mémoire dans le dernier : car notre trajet nous a entraîné d'une mémoire qui bloque l'adaptation (A) à une culture du risque dans une société assurantielle (B), en passant par une 
liaison étroite et structurée C), et une identité structurée par une mémoire orientée (D).

\section{A. Une mémoire qui bloque}

Dans le premier terrain, nous avions concentré notre attention théorique sur le phénomène de la mémoire. Loin de mobiliser, comme nous l'imaginions, la mémoire pouvait bloquer.

Une mémoire constituée, révérée, quasi sanctifiée est de celle qui fige un groupe dans des rites sinon dans des rituels. Ce type de mémoire se constitue généralement autour d'une origine prestigieuse, effaçant du même coup ce qui était là avant la fondation. Ce n'est donc pas une mémoire entière, mais une mémoire tronquée qui se présente comme toute la mémoire, celle qui tient lieu d'identité. C'est ce type de mémoire qui semble régir la micro-société du Sauze, lui donnant un aspect à la fois archaïque et féodal.

Archaïque, dans la mesure où l'événement qui lui a donné naissance est situé historiquement dans un temps dont aucun des paramètres n'a plus cours aujourd'hui. En préservant ce temps de la naissance comme facteur de l'identité actuelle, on se met à vivre au rythme et avec les valeurs, devenues obsolètes, de ce temps-là. Ce qui signifie, pour ce qui nous occupe ici, l'arrêt de toute investigation future. Le monde extérieur se referme, à la limite il n'existe même plus, il est nié, car il menace l'identité du système. Pour Le Sauze, Pra-Loup représente ce monde ouvert qui vit sur d'autres modèles et a adopté une autre temporalité. Il est naturel alors que Le Sauze essaie de regrouper sous son aile d'autres stations, petites et isolées, qui se constituent autour de l'image de la vallée d'Ubaye, " notre » vallée, avec ses mythes et sa mémoire propre. Isolée, aux dires des Ubayens eux mêmes, la vallée paraît entretenir cet archaïsme et s'en prévaloir. Ainsi les gens de la vallée n'acceptent-ils pas l'ouverture à ceux qu'ils considèrent comme des étrangers, fussent-ils même des montagnards.

Société féodale aussi, nous a-t-il semblé, parce que toute entière tissée par les liens qui unissent ses membres à l'héritier du fondateur, patron de l'entreprise qui fait vivre le pays. Il n'y a à Sauze qu'un seul pouvoir, celui-là. Le maire est absent, inexistant. Le maire-adjoint, lui, est très lié à la vallée de l'Ubaye et s'occupe de la mise en place du fameux contrat de pays; actif dans ce domaine, il est aussi lié au propriétaire de la station qui indirectement lui fournit des clients (le maire-adjoint est en effet aussi commerçant). L'exploration des ressources économiques et politiques qui seraient utiles pour sortir Le Sauze de l'impasse ne dépasse pas les limites du «pays », celles de la communauté des communes ; en aucun cas elle ne peut se comparer à l'activité ouverte du maire de Pra-Loup qui, par exemple, n'hésite pas à se rendre à la réunion des maires de stations de ski (il était justement à Paris pour une de ces réunions lors de notre enquête).

Le Sauze : un roi-patron, de fidèles et loyaux sujets serrés autour de la station, une volonté de fermeture qui a empêché la réalisation d'un projet considéré comme important : celui de construire le centre de la station à Super Sauze, à une altitude plus élevée, ce qui aurait permis une extension ; fermeture qui a empêché, de même, l'arrivée de nouveaux acheteurs en mettant le veto sur la vente des terrains. Telle est l'esquisse que nous 
pouvons tracer et dont la clef, nous l'avons dit, se trouve dans le type de mémoire constitutif de la station.

Pra-Loup : un équipe bien liée autour d'un maire actif, qui a des liens avec la région et le département sait monter ou faire monter des dossiers et les envoyer au bon endroit au bon moment. Qui anticipe le changement possible et ouvre le territoire à des investisseurs. Autrement dit une appréhension du temps souple, qui tient compte des aléas et se saisit des occasions.

Se délier de sa mémoire serait- il un moyen de se rendre adaptable ? Est-ce là une des conditions requises? Nous pensons que se délier de ce type de mémoire-là est nécessaire, cela ne touche pas d'autres sortes de mémoires et les autres moyens d'y faire appel.

Nous l'avons déjà précisé, la mémoire qui se bâtit à partir d'un événement fondateur déterminé, et uniquement à partir de là, est coupée de la grande mémoire, celle de toutes les mémoires plurielles qui composent le vaste panorama de la culture vivante. Les Grecs de l'Antiquité avaient un nom pour cette mémoire qui comprenait tout ce qui avait été accumulé dans les différentes strates : Mnémosyne, déesse de la mémoire. Cette mémoire-là est pour ainsi dire intemporelle puisqu'elle comprend tous les temps. Ainsi est-elle mémoire du présent, celui qui à chaque instant devient passé, et tisse ainsi un lien avec l'ensemble des temps.

\section{B. Culture du risque et sécurité assurantielle}

Dans le deuxième terrain l'exemple des SaintesMaries-de-la-Mer est venu renforcer ce résultat, en donnant un exemple similaire : cette ville qui s'accroche à la mémoire, même en dépit de la vie, confirme encore ce premier résultat. Mais aussi, en un autre sens, les difficultés redoublent : mémoire courte ou mémoire longue ? Si la mémoire courte peut bloquer, une mémoire plus longue pourrait-elle au contraire stimuler l'action?

Il y aurait ainsi en Camargue une tradition concernant les risques naturels, inondations et tempêtes, et la manière d'en éviter les conséquences. Les Anciens, nous dit-on, construisaient leur fermes sur les bourrelets du Rhône (les levées de terre que le Rhône avait laissées en changeant de lit) et se voyaient ainsi hors de portée des inondations. Plusieurs mas que nous avons visités et où sont installés le Parc Naturel Régional et la Réserve nationale : le mas de Rousty, de même que la Capellière ou le mas de Bélugue, suivent effectivement cette tradition. C'est que les inondations étaient fréquentes, le Rhône sortait souvent de son lit, les gens étaient habitués et se prémunissaient contre les dégâts possibles.

Comme souvent, ce sont les modes de construction, les matériaux employés, de même que les manières d'habiter- et aussi, naturellement les façons de cultiver la terre, les modes de gestion des réserves, etc. qui sont alors transmises. Dans ce cas, un tel ensemble fait « corps » autour d'un souci permanent qui dicte les conduites et les manières de faire : le souci du danger possible, la présence permanente des risques que l'on court. C'est du moins ce que laissent penser les discours actuels sur cette « culture », englobant dans celleci le type de constructions irrégulière des anciens mas dont les différents niveaux donnent sur 
des escaliers tortueux, où des celliers peu aérés conservent mal les vins, d'après M. Poulle ${ }^{3}$.

Dans la culture du risque, version Camargue, il ne s'agit pas du risque pour le risque. Il ne s'agit pas d' " oser », de prendre une décision pour gagner un but en comptant sur la chance, mais de préserver des conditions existantes, de subir le risque, de supporter les aléas et non de les provoquer...

C'est - dirions-nous - l'acceptation d'un « risque à l'ancienne ».

Traditionnellement, en effet, il était seulement question, pour les habitants de la Camargue, de vivre avec les conditions qui étaient celles du terrain et si celles-ci comportaient des risques, ces risques étaient acceptés au même titre que les inconvénients habituels qui accompagnent la vie des agriculteurs : la sécheresse ou la pluie. Pour cette acception, risque et vulnérabilité allaient de pair, et les aléas étaient identifiés comme risques à venir.

Tel est donc le sens de cette « culture du risque » qui nous avait étonnés au premier abord; elle correspond point par point au projet des environnementalistes : revenir à une nature, aménagée sans doute, mais souplement, conscience de la précarité des ressources, de la vulnérabilité du terrain et conscience des risques. C'est l'attitude qui est vue comme celle des anciens habitants de la Camargue, et il y a donc une sorte de cohérence à faire référence à cette culture, dans le cadre d'une protection de la nature (habitants, animaux et végétaux confondus) et de prôner en même temps le retour à une vie plus naturelle.
Pour autant, doit-on ignorer la notion moderne de risque qui, avec son antithèse, la sécurité, caractérise la société contemporaine ? Ici, la société technicienne prévoit alors des dispositions sécuritaires : assurances multiples, réassurées ellesmêmes et calculées selon les lois statistiques de probabilité. La sécurité forme désormais, avec le calcul du risque, le nouveau couple gestionnaire de la vie de chacun. C'est alors une prévention tout azimut qui prévaut, on s'assure du risque avant même de savoir quel il est. Ce qui est visé c'est l'état zéro du risque, poursuite utopique s'il en est, aussi utopique que l'idée de santé parfaite analysée par Lucien $\mathrm{Sfez}^{4}$ et porteuse d'une idéologie semblable : la sécurité, l'assurance tous risques, l'idéal du risque zéro...

Que devient alors cette culture du risque, basée sur la mémoire, qui devrait guider nos Camarguais et qu'ils devraient retrouver sous l'oubli qui l'a fait disparaître?

L'adaptabilité tient à la conscience d'une fragilité, à la perception d'un signal de détresse. La perception de la vulnérabilité est liée au sentiment de la fragilité de l'équilibre actuel, et sans doute aussi à une sorte de compassion pour un milieu en voie de disparition. En aucun cas la vulnérabilité dont dépend l'apprentissage de l'adaptation ne peut être ressentie quand on est sûr de soi, égocentré et totalement assuré et réassuré.

\section{Une liaison forte}

Dans la troisième enquête un autre phénomène apparaît, celui de la liaison, d'une liaison forte. Cette troisième enquête montre que le choix de mémoire doit être orienté fermement par 
une instance efficace, écoutée, intelligente, qui doit exercer une liaison entre les problèmes environnementaux et la population. Nécessité de cette liaison qui est exercée en Champagne par le C.I.V.C. Le C.I.V.C. est l'interlocuteur central et le véritable partenaire dans une négociation. Il sera très favorable à toute proposition pour une viticulture raisonnée, voire écologique sans excès. Sa position est centrale d'un point de vue stratégique puisque il coordonne l'activité technique et économique du champagne. N'oublions pas que le C.I.V.C. est présidé par deux présidents de rang égal et qui représentent le syndicat des vignerons et les grandes maisons de Champagne. C'est le C.I.V.C. qui structure la politique champenoise et la structurera de plus en plus.

La conduite désormais bien rôdée des minimes adaptations en Champagne tient à présent de la routine, mais elle a peut-être besoin d'être repensée à la lumière des récentes évolutions de l'écosystème. En effet, il n'est peut-être pas souhaitable de continuer à produire autant et de la même manière (même si la production intensive des années 50 a été abandonnée). Nappes phréatiques polluées et sol épuisé pourraient bien être le résultat de ces pratiques, comme le dénoncent les « bios ». C'est pour avoir négligé ce point et s'être contentés de continuer à exploiter leurs ressources dans les mêmes mesures et avec les mêmes outils que des sociétés entières ont disparu. «Qu'a pu penser le Pascuan qui a abattu le dernier arbre de son île? » se demande Jared Diamond ${ }^{5}$. Sans aller jusqu'à cet exemple extrême, on peut constater qu'il y a dans l'euphorie d'une récolte sans précédent, une sorte d'aveuglement sur les conséquences d'une telle surproduction.
Or ce n'est qu'en reliant la question des gaz à effets de serre au problème du changement climatique qu'un pas décisif peut être fait pour l'adaptation. Aussi bien dans la mise au point de techniques nouvelles destinées à enrayer la production des émissions, que dans la construction et la mise en circulation d'engins bi-énergies et de tracteurs d'une nouvelle conception (pouvant " enjamber » les rangs de vigne, par exemple). Mais la liaison de ces deux questions est justement ce qui fait défaut la plupart du temps, et c'est là un obstacle à une adaptation réussie. Cette conscience de la liaison entre les deux phénomènes faisait aussi défaut dans nos enquêtes précédentes en particulier à Le Sauze et aux Saintes- Maries.

Si notre analyse de la situation en Champagne permet de penser que les conditions d'une adaptation réussie sont à peu près réunies, il n'en reste pas moins que le passage à l'acte n'est pas évident, il y faut encore une bonne appréhension et des menaces et des solutions possibles. Ces conditions favorables étaient réunies aussi à PraLou. Mais l'ultime condition est-elle remplie dans un cas comme dans l'autre?

La perception du danger n'est pas toujours aisée, et la capacité de le déceler tient aux liaisons entre des événements apparemment étrangers les uns aux autres et à l' évaluation que l'on peut en faire.

\section{L'identité structurée par une mémoire orientée}

La quatrième enquête a porté sur le bordeaux, car nous savions que d'une part le C.I.V.B. était 
moins fort, moins structuré que le C.I.V.C., et donc qu'une politique d'innovation aurait plus de mal à passer dans une telle instance qu'au C.I.V.C., et que, d'autre part, la crise économique était passée par là, et qu'elle avait toute priorité dans les consciences sur tout autre problème, même s'il s'agit d'urgence écologique, et alors même que la crise sans avoir disparu, s'est estompée.

Cette quatrième enquête nous a conforté dans l'idée d'une liaison nécessaire et a mis en avant un devoir de communication renforcée, d'orientation forte et d'impulsion centralisée qui ne peut venir que d'une instance professionnelle. Quand celle-ci fait défaut, l'effondrement menace $^{6}$. Cette fois-ci, avec la mémoire qui freine comme à Sauze et aux Saintes-Maries-dela-Mer, nous trouvons le problème de l'identité. On se replie sur l'identité contre des concurrents étrangers qui font un vin industriel, sans typicité, on revendique son histoire et son particularisme fort face à un changement aussi bien climatique que social.

Autant le C.I.V.C. est une structure robuste et unie, sans exclure, bien sûr, des tensions internes, autant le C.I.V.B. est une structure molle, parfois incapable de prendre des décisions. Négociants et vignerons se font contrepoids jusqu'à s'annuler les uns les autres. Quel est le noyau dur au C.I.V.B. ? Sa structure administrative, c'està-dire son directeur général et ses commissions techniques dotées de directeurs compétents. Ce n'est pas rien, mais cette structure administrative ne saurait remplacer une entente constructive et de longue durée entre vignerons et négociants, ni tenir lieu, à elle seule, de politique.
Résultat de ces indécisions : l'attitude envers le réchauffement et les menaces sur la planète est totalement différente dans les deux cas.

En mettant l'accent sur cette fragilité, l'analyse du cas bordelais nous conduit à approfondir notre vision de l'adaptation en traitant les rapports entre mémoire et identité. Mais comment traiter le conflit entre une identité revendiquée et l'obligation d'une standardisation due à la mondialisation ? Le rapport entre mémoire et identité doit être entièrement repensé. Plus encore : si la mondialisation exige un changement d'échelle dans les problèmes de l'identité, un changement de plus grande envergure qui affecte la planète et menace la survie humaine, le changement climatique, fait nécessité d'adopter une stratégie de mémoire entièrement différente : au lieu de faire revivre des éléments du passé, chronologiquement (par exemple, le passé glorieux de Bordeaux, sa fameuse place de marché, ses stars du vin), c'est une mémoire orientée qu'il faut développer.

Qu'est ce qu'une mémoire orientée ? Pour en avoir une idée, observons les scénarios du futur. Il s'agit là de stratégie prospective. Les scénarios, on le sait, jouent un rôle important dans la vulgarisation des résultats scientifiques concernant le changement climatique : scénario du « 2 degrés en plus d'ici $2030 »$, scénario plus pessimiste du « 4 à 6 degrés en plus », scénario catastrophique comme celui de M. Jancovici qui tire son impact des précisions qu'il donne et des illustrations schématiques qui les accompagnent. Outre les qualités indiscutables de la méthode des scénarios que l'on connaît depuis les années 50, la possibilité de mettre ces scénarios en cartes, en schémas 
et même en images est un atout important; cela permet d'imaginer des séquences alternatives à partir de situations données, et surtout de les vulgariser aisément, car les images-chocs de futurs possibles sont plus «parlantes » qu'une accumulation d' arguments.

Que manque-t-il alors pour que la méthode des scénarios prenne sur ce terrain?

En réalité, la carence de la communication dont les Bordelais se plaignent si fort, n'affecte pas seulement le marché, comme ils le croient, elle manque à l'orientation de la mémoire. En effet, une étape importante des scénarios prospectifs est celle qui, suivant le recueil d'informations et l'élaboration des modèles, consiste à les rendre utilisables, en les expliquant au public. Ce chaînon de communication manquant grève les modestes essais d'anticipation, ainsi que le désir de s'adapter, pourtant présent. Car un projet d'adaptation ne peut être entrepris seul, il doit s'inscrire dans un dessin global, voire un dessein nettement exprimé, dont on peut reconnaître les contours et qu'on peut s'approprier. Ainsi, autant les adaptations ponctuelles au climat local sontelles omniprésentes chez les viticulteurs, autant une adaptation plus large et significative d'un changement fait-elle défaut. C'est que le viticulteur, travaillant avec le climat, le sol, et les cépages, suit « naturellement » l'évolution de ces trois composantes, s'adaptant au fur et à mesure des événements climatiques, tout comme la vigne elle même s'adapte « naturellement », et même si ce naturel n'a rien de naturel, le végétal et les procédés humains qui améliorent son développement marchent du même pas. Toujours s'adapter, au coup par coup, tel est l'axiome de base.
Mais c'est tout autre chose quand il ne s'agit plus de nature, mais de société, non d'actes ponctuels mais de globalité, non de phénomènes maîtrisables à plus ou moins court terme, mais de phénomènes incertains dans le temps, non cernables, et qui demandent des prévisions à une ou deux générations...

Or les problèmes de différenciation, et les revendications d'identité ne peuvent se traiter et encore moins se résoudre en dehors d'un plan général d'action qui oriente les actions particulières.

C'est un tel plan et sa publicisation qui manquent pour une adaptation des identités (individuelles ou de groupes) à un environnement de plus en plus global et complexe.

Nous aimerions donc insister, après cette enquête, sur ce dernier point : une structure objective de liaison est nécessaire pour orienter la mémoire et donner forme à l'identité, que celle-ci soit individuelle ou collective. Il n'y a pas d'identité per se. De même n'y a-t-il pas de mémoire en soi. La mémoire est toujours orientée par le projet qui la sous-tend. On se souvient pour quelque chose, sinon la mémoire tourne sur elle même, prise dans un ressassement souvent amer. C'est le projet qui la rend vivante, tout comme l'identité ne peut se construire que par rapport à l'autre. Terroir, typicité, identité et même « nature » sont des constructions patientes, qui ont besoin d'être soutenues et qu'un changement de société peut mettre à mal.

L'ethnologie et l'histoire nous l'ont enseigné : faute d'une stratégie de liaison assez forte, et faute d'anticipation raisonnée beaucoup de 
sociétés ont disparu, ruinées par les divisions internes et par l'entêtement dans le culte de soi.

En conclusion, les facteurs que l'on croit positifs, tels que mémoire ou identité, se révèlent des obstacles quand ils ne sont pas orientés, quand une communication vectorisée ne s'établit pas entre les habitants, les professions, le pouvoir local et national et les questions environnementales plus larges. Une stratégie globale d'adaptation doit tenir compte fondamentalement d'une communication orientée entre tous ces points.
$\mathrm{N} \cdot \mathrm{O} \cdot \mathrm{T} \cdot \mathrm{E} \cdot \mathrm{S}$

1. Attitudes face à l'adaptation au changement climatique : le cas de deux stations de moyenne montagne dans les Alpes de Haute-Provence, par Lucien Sfez et Anne Cauquelin, Credap/Credatic, 2005. Attitudes face à l'adaptation au changement climatique : le cas de la Camargue, par Lucien Sfez et Anne Cauquelin, Credap/Credatic, 2006. Attitudes face à l'adaptation au changement climatique : le cas $d u$ Champagne, par Lucien Sfez et Anne Cauquelin, Credap/Credatic, 2007. Attitudes face à l'adaptation au changement climatique : le cas du Bordelais, par Lucien Sfez et Anne Cauquelin, Credap/ Credatic, Juin 2008.

2. Contact pour obtenir les rapports : Mme Derkenne, $<$ chantal.derkenne@ademe.fr>

3. M. Poulle, Étude de la Camargue ou statistiques du delta du Rhône, envisagée principalement sous le rapport des améliorations dont il est susceptible, Arles, 1927, cité par Bernard Picon, L'espace et le temps en Camargue, Actes sud, 1988.

4. Lucien Sfez, La Santé parfaite, Le Seuil, 1995. La santé parfaite qui était décrite, c'était la santé parfaite de l'individu dans la santé parfaite de la planète.

5. Nous faisons allusion ici à l'ouvrage, fondamental pour notre propos, de Jared Diamond : Effondrement ou comment les sociétés décident de leur disparition ou de leur survie, Gallimard, 2006.

6. Voir Jared Diamond, Effondrement ou comment les sociétés décident de leur disparition ou de leur survie, op.cit. 
$R \cdot E ́ \cdot S \cdot U \cdot M \cdot E ́$

On présente ici quatre cas d'adaptation au changement climatique : deux villages sans neige dans les Alpes du Sud, divers micros lieux envahis par les eaux en $\mathrm{Ca}-$ margue, le cas des vignobles de Champagne, le cas des vignobles bordelais. Or la résistance au changement climatique est chaque fois différente. Les structures locales jouent un rôle déterminant dans la prise en compte ou le refus de l'adaptation au changement climatique.

Here, there is four cases of adaptation to the climatic change : two villages without snow in the Alpes du Sud, some micros places invaded by waters in Camargue, the cases of vineyards of Champagne, the case of vineyards in Bordeaux region. Therefore, the resistance to adaptation to the climatic change is different each time. The local structures have a decising part to refuse or to accept adaptation to the climatic change . 